\title{
Risk Free Simulated Environment to Assess Colorectal Fellow Technical Skills: A Pilot Study
}

\author{
AlJ amal Y*, Mathis K and Dozois E \\ Division of Colon and Rectal Surgery, Mayo Clinic, USA \\ *Corresponding author: Yazan AlJ amal, Division \\ of Colon and Rectal Surgery, Mayo Clinic, Rochester, \\ Minnesota, 200 First S SW, Rochester, MN 55905, USA
}

Received: December 01, 2017; Accepted: December 22, 2017; Published: December 29, 2017

\begin{abstract}
Introduction: Evaluation of residents' surgical skill by teaching faculty is generally a subjective assessment done at the completion of a rotation and based on recollection of resident performance. This type of assessment has been shown to have poor reliability and validity and is often determined by factors other than technical skill. To test whether or not we could develop an objective assessment process for technical competency in a risk-free environment, we developed a protocol that utilized a cadaver model to assess operative performance.
\end{abstract}

Methods: To determine whether objective assessment in the cadaver lab would correlate with assessments in the clinical setting, we developed a standardized assessment tool that would be consistently used by faculty to assess operative skills. 5 operations were selected for assessment; open and laparoscopic right and left colectomy for cancer and open proctectomy for cancer. A baseline assessment was done within one month of entering the fellowship and then at the mid-point and end of the fellowship year. Operations performed in the cadaver lab were un-coached and operatives performed in the clinical setting were assisted by faculty at their discretion. Timing of the cadaver labs correlated with the clinical assessment times to match skill set assessment along the year timeline.

Results: Three fellows completed all required operations and underwent standardized objective assessments. The fellows' mean scores in the five different operations at the three separate time frames were $72,94,100$ for laparoscopicright-hemicolectomy, 64,89,100 for laparoscopic-left-hemicolectomy, 71,99,100 for open-right-hemicolectomy, $71,98,98$ for open-left-hemicolectomy, and $68,95,100$ for LAR compared to their results in the clinical setting $87,97,100 \_68$ ,92,99_78,99,100_86,98,98 and 80,95,100 subsequently.

Conclusion: This pilot study demonstrates the feasibility of utilizing cadavers in a risk-free simulated OR for objective clinical skills assessment. Fellows' technical skills improved throughout the course of the year and demonstration of this improvement was found in both the clinical and simulated environment. The cadaver model, in addition to an objective assessment tool, appears to be a good surrogate for skills performance competence in the clinical setting.

\section{Introduction}

Board certified in general surgery required a completion of an accredited 5-year program, pass the written board exam and meet all the requirements by their program and be certified by the program directors as competent and qualified [1]. While residency program directors have different tools to evaluate trainees, the end rotation evaluations by the staffs are the most common evaluation approach [2]. This type of evaluation limits the ability to provide a meaningful feedback to the surgical resident regarding their surgical competency. This type of assessment has been shown to have poor reliability and validity [3] and is often determined by factors other than technical skill.

Surgical trainee competence has traditionally been linked to surgical volume [4]. Residency review committees and certifying boards use case volume experience as a benchmark of resident competence to safely perform surgical procedures independently. With the implementation of the 80 work hour restriction in July 2003 , surgical case volume and operative experience among graduating residents has declined. The risk of producing and graduating inferior chief residents is real in general surgery.

Cadaver-Based Simulation is beneficial for all residents as operative autonomy and independence in the operating room which are crucial components to the transition of chief residents to independently practicing surgeons are improved [5-10]. Also, there has been measured erosion in resident confidence to operate independently $[8,9]$. Using Cadaver is assessing the surgical competency among the surgical resident has been addressed.

Because of the uncertainty of baseline technical competence of graduating residents entering our colon and rectal surgery fellowship, and to test whether or not we could develop an objective assessment 

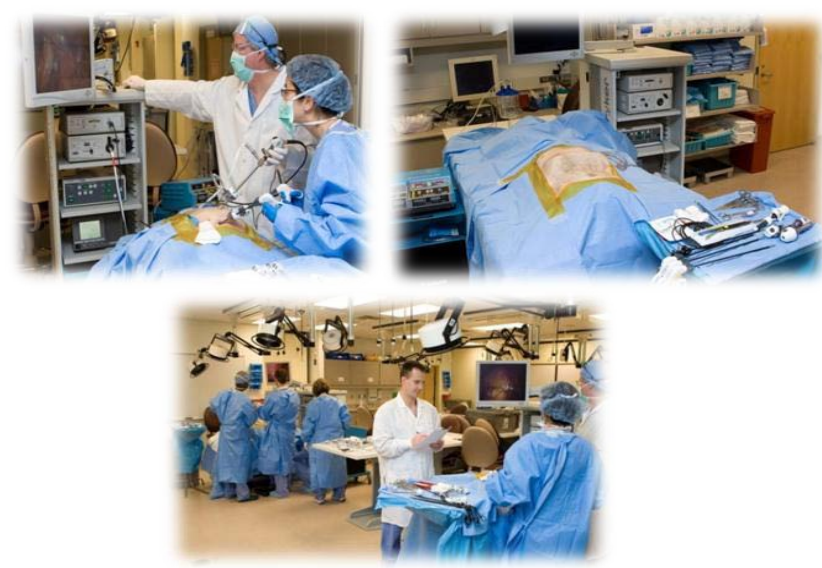

Figure 1: Cadaver Lab (A, B and C).

process for technical competency in a risk-free environment, we developed a protocol that utilized a cadaver model to assess operative performance.

\section{Methods}

Three ACGME colon and rectal fellows participated in our pilot study. Technical competency assessments were done in two settings; 1 ) in the cadaver lab using simulated OR environment 2) clinical setting as part of the fellowship program. Fellows' performed complete open and laparoscopic operations on the cadavers completely independent with no coaching. Assessments of similar operations were done in the clinical setting and assistance during the operation was at the discretion of the faculty. A standardized, objective assessment form was used to score each operation (Objective Structural Assessment of Technical Skills forms, 2017). Fellows were assessed at the beginning of the fellowship year, midway through and at the end of training. Assessments in the cadaver lab and in the clinical setting were done within a narrow timeline of each other so that competency in both settings could be compared at the same point throughout the year.

The fellows completed all required operations and underwent standardized objective assessments. Each fellow performed the following five operative procedures on fresh frozen cadavers (Figure 1) and the clinical setting (Figure 2): laparoscopic-assisted right colectomy without an anastomosis, laparoscopic-assisted or handassisted (fellow's choice) left colectomy without an anastomosis, open right colectomy with stapled anastomosis, open left colectomy with hand-sewn two-layer colorectal anastomosis, and a pelvic dissection with proctectomy and total mesorectal excision (Figure 3). Each operation was performed with the assistance of volunteer operating room staff and general surgery residents. No coaching was allowed or performed. One of two consultant surgeons evaluated each fellow on each procedure (Figure 1). Fellows received immediate feedback following each operation.

\section{Results}

All CRS fellows completed their assessment in both cadaver lab and clinical setting at baseline, 6 month (mid-point) period and at the end of their fellowship. The cadaver lab assessments were completed over two days and required two cadavers per fellow; the approximate
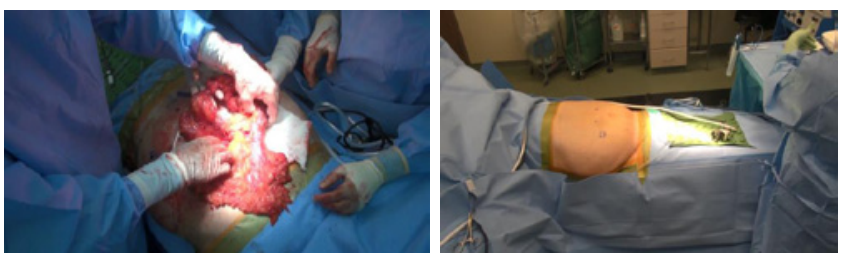

Figure 2: Clinical setting

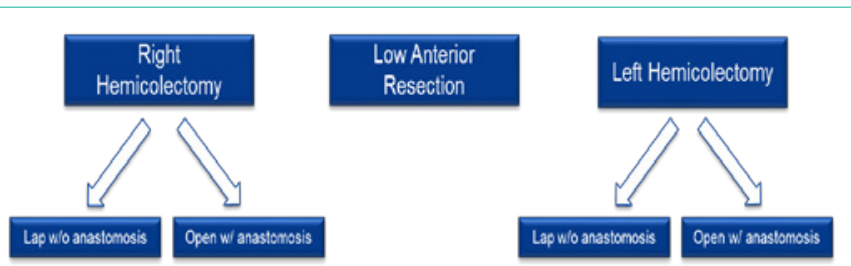

Figure 3: Five operative procedures.

cost of the endeavor was $\$ 6600$ for the baseline evaluation (and $\$ 6600$ for each of the 2 subsequent evaluations).

The clinical assessments were completed within one month of the cadaver lab assessments and the fellows' schedule. All CRS Fellows' baseline, 6 month (mid-point) period and at the end of their fellowship scores at both cadaver lab and clinical setting in open right colectomy, open left colectomy, laparoscopic-assisted right colectomy, laparoscopic-assisted left colectomy, and low anterior resection are summarized in (Table 1). Significant improvements ( $\mathrm{p}$ value $<0.05)$ in the overall competency scores were seen at 6 and 12 months in both the cadaver lab and clinical setting in all five procedures (Figure 4). There was close correlation in the cadaver lab and clinical setting scores at the three different assessments time points. (Table $1 \&$ Figure 4).

\section{Discussion}

In this pilot study we aimed to test whether or not we could develop a process to objectively assess technical competency in colon and rectal fellows using a risk-free environment. Utilizing cadavers in a simulated operating room, we were able to demonstrate that assessment in this setting was a good surrogate for how trainees performed in the clinical setting. Fellow scores improved over the course of the year and assessments in the cadaver lab correlated very closely with assessments in the clinical setting during similar time points during a 1 year fellowship.

Objective assessment of technical skill is essential during surgical training for formative feedback and to determine specific milestone achievement. At the completion of training, program directors must attest as to whether a trainee is competent to practice independently. Because trainees have few opportunities to perform operations without the assistance of faculty, how trainees would perform in an un-coached operation may not truly be known until they enter practice. If a risk-free environment could be developed to allow trainees to complete entire operations independently and then be scored on performance using predetermined outcome measures, it would be ideal for feedback, focused learning and summative evaluations. 


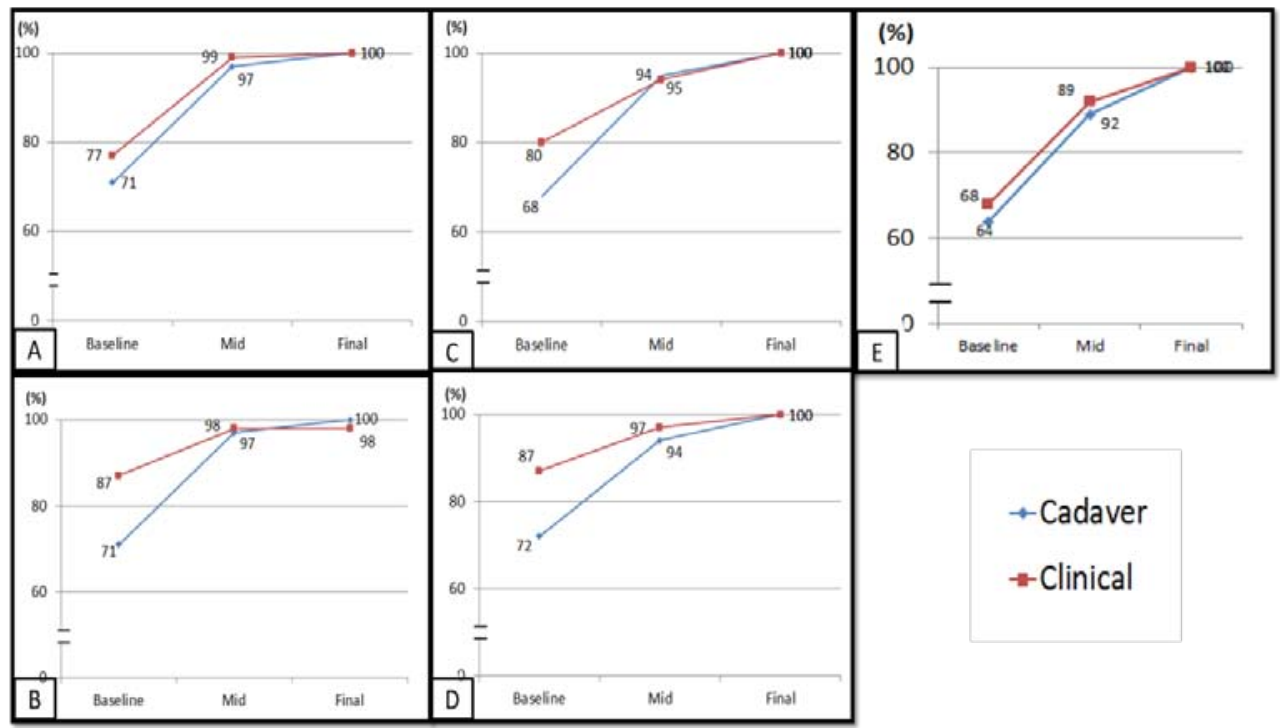

4: A) Open right hemicolectomy; B) Open left hemicolectomy; C) Low anterior resection; D) Lap right hemicolectomy; E) Lap left hemicolectomy.

Table 1: Summary of CRS Fellow's mean scores in 5 procedure at both Cadaver and clinical.

\begin{tabular}{|c|c|c|c|c|c|c|c|c|c|c|c|c|c|c|c|c|c|c|c|c|c|c|c|c|}
\hline & \multicolumn{8}{|c|}{ Baseline } & \multicolumn{8}{|c|}{ Mid-point } & \multicolumn{8}{|c|}{ Final } \\
\hline & \multicolumn{4}{|c|}{ Cadaver } & \multicolumn{4}{|c|}{ Clinical } & \multicolumn{4}{|c|}{ Cadaver } & \multicolumn{4}{|c|}{ Clinical } & \multicolumn{4}{|c|}{ Cadaver } & \multicolumn{4}{|c|}{ Clinical } \\
\hline & $\mathrm{F} 1$ & $\mathrm{~F} 2$ & F3 & Mean & $\mathrm{F} 1$ & $\mathrm{~F} 2$ & F3 & Mean & $\mathrm{F} 1$ & $\mathrm{~F} 2$ & F3 & Mean & F1 & $\mathrm{F} 2$ & F3 & Mean & $\mathrm{F} 1$ & $\mathrm{~F} 2$ & F3 & Mean & $\mathrm{F} 1$ & $\mathrm{~F} 2$ & F3 & Mean \\
\hline $\begin{array}{c}\text { Laparoscopic } \\
\text { Right Colectomy }\end{array}$ & 70 & 77 & 70 & 72 & 72 & 94 & 94 & 87 & 97 & 89 & 97 & 94 & 100 & 97 & 94 & 97 & 100 & 100 & 100 & 100 & 100 & 100 & 100 & 100 \\
\hline $\begin{array}{l}\text { Laparoscopic } \\
\text { Left Colectomy }\end{array}$ & 67 & 63 & 61 & 63 & 69 & 89 & 72 & 68 & 89 & 82 & 91 & 89 & 94 & 93 & 94 & 92 & 100 & 97 & 100 & 99 & 100 & 100 & 100 & 100 \\
\hline $\begin{array}{l}\text { Open Right } \\
\text { Colectomy }\end{array}$ & 67 & 79 & 67 & 71 & 70 & 87 & 75 & 77 & 92 & 97 & 100 & 97 & 100 & 100 & 100 & 99 & 100 & 97 & 100 & 99 & 100 & 100 & 100 & 100 \\
\hline $\begin{array}{l}\text { Open Left } \\
\text { Colectomy }\end{array}$ & 67 & 79 & 67 & 71 & 75 & 93 & 97 & 87 & 92 & 100 & 100 & 97 & 97 & 97 & 100 & 98 & 97 & 100 & 100 & 99 & 100 & 97 & 100 & 99 \\
\hline $\begin{array}{l}\text { Low Anterior } \\
\text { Resection }\end{array}$ & 59 & 63 & 67 & 68 & 80 & 78 & 97 & 80 & 96 & 93 & 93 & 95 & 97 & 96 & 93 & 94 & 100 & 100 & 100 & 100 & 100 & 100 & 100 & 100 \\
\hline
\end{tabular}

High fidelity simulators have been developed, and are good for specific skill assessments, but no simulator to date has been developed that allows a trainee to perform an entire complex operation like a colectomy [11]. Human cadavers offer the best simulation to real surgery but are a limited resource and costly [12]. Animal models have also been used for specific task assessment but limitations include availability, cost and inability to simulate human anatomy.

Several methods of assessment of surgical skill have been described in the literature [13]. Despite literature strongly supporting objective assessment through standardized assessment tools, most assessments are documented in an evaluation form filled out at the end of a rotation. These evaluation forms often combine other assessments such as knowledge and professionalism with technical competency. These evaluation forms, often filled out days to weeks after the rotation ends, relies heavily on faculty recollection of how the trainees performed in various procedures. This type of assessment has shown poor reliability and validity [1-3]. The specific technical competency assessment process is feasible and provides an objective assessment of the trainees' surgical competency. It provides our incoming CRS fellows, a unique opportunity to perform complex surgical operations with no coaching, immediate feedback with hands-on instruction with highlighting areas for individual improvement. It allows for easy accessibility, is ideal for teaching, and widespread usage is possible in academic settings.

Limitations to this study include the small number of fellow assessments, use of multiple faculties for assessments and patient variables. Given that this was a pilot study, we were mainly interested in feasibility of carrying out simulated operations in the cadaver lab. It is worth mentioning also that significant barriers may exist in expanding this type of technical assessment process across fellowship programs. Resources in terms of cadavers and equipment and cost may be significant barriers. In addition, excusing faculty and trainees from their usual duties may be challenging when time away from clinical duties is difficult to come by and requires colleague and institutional support.

In summary, this pilot study utilizing cadavers in a simulated operating room, demonstrated that assessment in this setting was a good surrogate for how trainees performed in the clinical environment. Trainee assessments in the cadaver lab correlated very closely with assessments in the clinical setting during similar time points during a 1 year fellowship. Further investigation using a risk-free environment like the one in this study is necessary to see if this approach to technical competency assessment is worth the investment in time and money. If a cadaver-based simulation of 
operative skill proves to be a good surrogate for clinical performance, it could be used to assess surgical milestone achievement, provide clear and objective feedback and potentially be used for a high stakes exam as part of a board certification process [14].

\section{References}

1. Jackson JL, Kay C, Frank M. The validity and reliability of attending evaluations of medicine residents. SAGE Open Med. 2015; 3: 2050312115589648.

2. Chaudhry SI, Holmboe E, Beasley BW. The state of evaluation in internal medicine residency." J Gen Intern Med. 2008; 23: 1010-1015.

3. Durning SJ, Cation LJ, Jackson JL. The reliability and validity of the American Board of Internal Medicine Monthly Evaluation Form. Acad Med. 2003; 78 1175-1182.

4. Bhatti $\mathrm{NI}$, Ahmed $\mathrm{A}$, Choi SS. Identifying quality indicators of surgical training A national survey. Laryngoscope. 2015; 125: 2685-2689.

5. De Montbrun SL, Roberts PL, Lowry AC, Ault GT, Burnstein MJ, Cataldo PA, et al. A novel approach to assessing technical competence of colorectal surgery residents: the development and evaluation of the Colorectal Objective Structured Assessment of Technical Skill (COSATS). Ann Surg. 2013; 258 1001-1006.

6. McLemore EC, Harnsberger CR, Broderick RC, Leland H, Sylla P, Coker AM, et al. Transanal total mesorectal excision (taTME) for rectal cancer: a training pathway. Surg Endosc. 2016; 30: 4130-4135.

7. Kim SC, Fisher JG, Delman KA, Hinman JM, Srinivasan JK, et al. CadaverBased Simulation Increases Resident Confidence, Initial Exposure to Fundamental Techniques, and May Augment Operative Autonomy. J Surg Educ. 2016; 73: 33-41.
8. Fonseca AL, Reddy V, Longo WE, Gusberg RJ. Graduating general surgery resident operative confidence: perspective from a national survey. J Surg Educ. 2014; 190: 419-428.

9. Bucholz EM, Sue GR, Yeo H, Roman SA, Bell RH, Sosa JA. Ourtrainees' confidence: results from a national survey of 4136 US general surgery residents. Arch Surg. 2011; 146: 907-914.

10. Kim SC, Fisher JG, Delman KA, Hinman JM, Srinivasan JK. Cadaver-Based Simulation Increases Resident Confidence, Initial Exposure to Fundamental Techniques, and May Augment Operative Autonomy. Journal of Surgical Education. 2016; 73: 33-41.

11. LeBlanc F, Champagne BJ, Augestad KM, Neary PC, Senagore AJ, Ellis $\mathrm{CN}$, et al. A comparison of human cadaver and augmented reality simulator models for straight laparoscopic colorectal skills acquisition training. J Am Coll Surg. 2010; 211: 250-255.

12. Eaton BD, Messent DO, Haywood IR. Animal cadaveric models for advanced trauma life support training. Ann R Coll Surg Engl. 1990; 72: 135-139.

13. Martin JA, Regehr G, Reznick R, MacRae H, Murnaghan J, Hutchison C, et al. Objective structured assessment of technical skill (OSATS) for surgical residents. Br J Surg. 1997; 84: 273-278.

14. de Montbrun SL, Roberts PL, Lowry AC, Ault GT, Burnstein MJ, Cataldo PA, et al. A Novel approach to assessing technical competence of colorectal surgery residents. Annals of Surgery. 2013; 258: 1001-1006.
Austin J Surg - Volume 4 Issue 5 - 2017

ISSN : 2381-9030 | www.austinpublishinggroup.com

AlJamal et al. (C) All rights are reserved
Citation: AlJamal Y, Mathis K and Dozois E. Risk Free Simulated Environment to Assess Colorectal Fellow Technical Skills: A Pilot Study. Austin J Surg. 2017; 4(5): 1118. 